\title{
Handheld optical coherence tomography device for photodynamic therapy
}

\author{
WANG TianShi ${ }^{1 \dagger}$, WANG ChengMing ${ }^{1 \dagger}$, HUANG NaiYan ${ }^{2}$, ZHANG Jian ${ }^{3}$, GU Ying $^{2 *} \&$ \\ XUE Ping ${ }^{1 *}$ \\ ${ }^{1}$ State Key Laboratory of Low-dimensional Quantum Physics and Laboratory of Atomic and Molecular Nanosciences, Department of Physics, \\ Tsinghua University, Beijing 100084, China; \\ ${ }^{2}$ Department of Laser Medicine, Chinese PLA General Hospital, Beijing 100853, China; \\ ${ }^{3}$ School of Electronic Engineering, Tianjin University of Technology and Education, Tianjin 300222, China
}

Received April 16, 2011; accepted June 9, 2011

\begin{abstract}
Photodynamic therapy (PDT) is a very effective treatment for port wine stains (PWS). To guide and assess PDT treatment, a handheld optical coherence tomography (OCT) probe was designed for real-time imaging of the PWS patient. The system uses a light source with a center wavelength of $1310 \mathrm{~nm},-3 \mathrm{~dB}$ bandwidth of $90 \mathrm{~nm}$ and an optical power of $9 \mathrm{~mW}$. The system also has a spatial resolution of $8 \mu \mathrm{m}$ (lateral) $\times 7 \mu \mathrm{m}$ (axial), an imaging rate of 4 frames per second, and a $102 \mathrm{~dB}$ sensitivity. We then demonstrate that the OCT imaging system can clearly distinguish between normal and PWS tissues. Therefore, the system can provide valuable guidance for PDT treatment.
\end{abstract}

optical coherence tomography, photodynamic therapy, port-wine stain

Citation: Wang T S, Wang C M, Huang N Y, et al. Handheld optical coherence tomography device for photodynamic therapy. Chin Sci Bull, 2012, 57: 450-454, doi: 10.1007/s11434-011-4864-6

A port-wine stain (PWS) is a vascular birthmark consisting of superficial and deep dilated vascular capillaries in the skin, which produces a reddish to purplish discoloration of the skin. They are named for their color, which resembles that of port wine. It is part of the family of disorders known as vascular malformations. Photodynamic therapy (PDT) is widely used in port-wine stain treatment [1-4]. First, patients are injected with a photosensitizer, and then exposed to laser radiation. The laser dissociation product of the photosensitizer destroys the capillary in the lesion without any damage to the skin. Therefore, PDT is a very effective technique and is widely used to cure PWS. A variety of photosensitizers have been studied [5-7]. Recently, multi-photon spectroscopic behaviors and nanostructures have been shown to affect the performance of the photosensitizer and attracted much attention [8-10]. However, because of the

These authors contributed equally to this work

*Corresponding authors (email: guyinglaser@sina.com; xuep@tsinghua.edu.cn) lack of real-time surgical guidance and noninvasive treatment assessments, PDT treatments are generally conservative, and the dose of laser exposure is primarily based on the doctor's previous experience. Although a laser Doppler rheometer can provide the mean velocity and the concentration of moving red blood cells in the superficial skin layers, it provides no depth information and fails to show the skin structure. This is crucial in determining the appropriate laser dose. Ultrasound imaging can supply the depth-resolved structure information, but its spatial resolution is too low for general PWS diagnosis in clinics. The diameters of the vascular capillaries of a PWS patient are typically between 10 and 100 microns. However, the resolution of ultrasound imaging is only on the millimeter or sub-millimeter scale. Biopsies can provide morphological information for skin, but they are invasive and cannot be done in real time. This treatment option is unacceptable for facial PWS patients because the biopsy may create a scar on the face of the patient. Therefore, a noninvasive and real-time cross-sectional 
imaging technique with high spatial resolution is needed for clinical diagnosis and surgical guidance.

Optical coherence tomography (OCT) is a new technique for in vivo and real time biomedical imaging with micronscale resolution $[11,12]$. The OCT is similar to ultrasound B-mode imaging, but OCT uses light rather than sound. OCT is an optical signal acquisition and processing technique. OCT is based on a low coherence interferometer technique. OCT can achieve extremely high-quality and high sensitivity imaging with resolutions 10-100 times higher than conventional ultrasound imaging, magnetic resonance imaging or computed tomography [13]. The imaging depth for skin is limited by optical attenuation and scattering from bio-tissue. Consequently, this results in depths limited to several millimeters. However, this imaging depth is enough for PWS diagnosis and treatment because the vascular malformations of PWS occur at the papillary layer. This layer is less than $1 \mathrm{~mm}$ under the surface. Therefore, in this paper we focus on the technical demonstration of an OCT system that uses a handheld probe. This will show the viability of the system for the real-time imaging of PWS. The system can be used in clinics because it is compatible with other current technologies in hospitals and meets all the requirements enforced for surgical treatments.

\section{System}

The handheld OCT is shown in Figure 1. A low coherence (or broadband) light beam is split into two arms which are sample and reference arms. The combination of the reflected light from the sample and the reference arm can generate interferential fringes if the optical path length difference for the two arms is less than the coherence length of the light source. While a rapid scanning optical delay (RSOD) [14] line in the reference arm scans, the demodulation of the interference fringes provides a depth-resolved sample signal. A cross-sectional image can be obtained by scanning along the sample surface. In our OCT system, a super luminescent diode (SLD), operating at $1310 \mathrm{~nm}$ with a spectral width of $90 \mathrm{~nm}$ and output optical power of $5 \mathrm{~mW}$, is used as the light source. Also, an optical circulator (in addition to a $2 \times$ 2 coupler) is used to improve the signal gain. The light from port 2 of the system is split into two parts using a $1 \times 2$ coupler. To achieve the best sensitivity, the ratio of power in the two arms is optimized. In this case, we coupled $80 \%$ of the power into the sample arm and $20 \%$ into the RSOD. The interference signal is detected, amplified and demodulated using a photo detector and a low-noise band-pass amplifier. The signal is digitized using an A/D card at a sampling rate of $10 \mathrm{MHz}$. Using the same card, the two driving signals are sent to scanners in the RSOD and handheld probe to synchronize all the sampling and scanning processes. An imaging speed of 4 frames per second and an array of $400 \times$ 400 pixels were implemented to fulfill the medical imaging needs. The spatial resolution is $8 \mu \mathrm{m}$ (lateral) $\times 10 \mu \mathrm{m}$ (axial) and the sensitivity can be as high as $102 \mathrm{~dB}$. Because the index of the tissue is about 1.35 and the axial resolution is

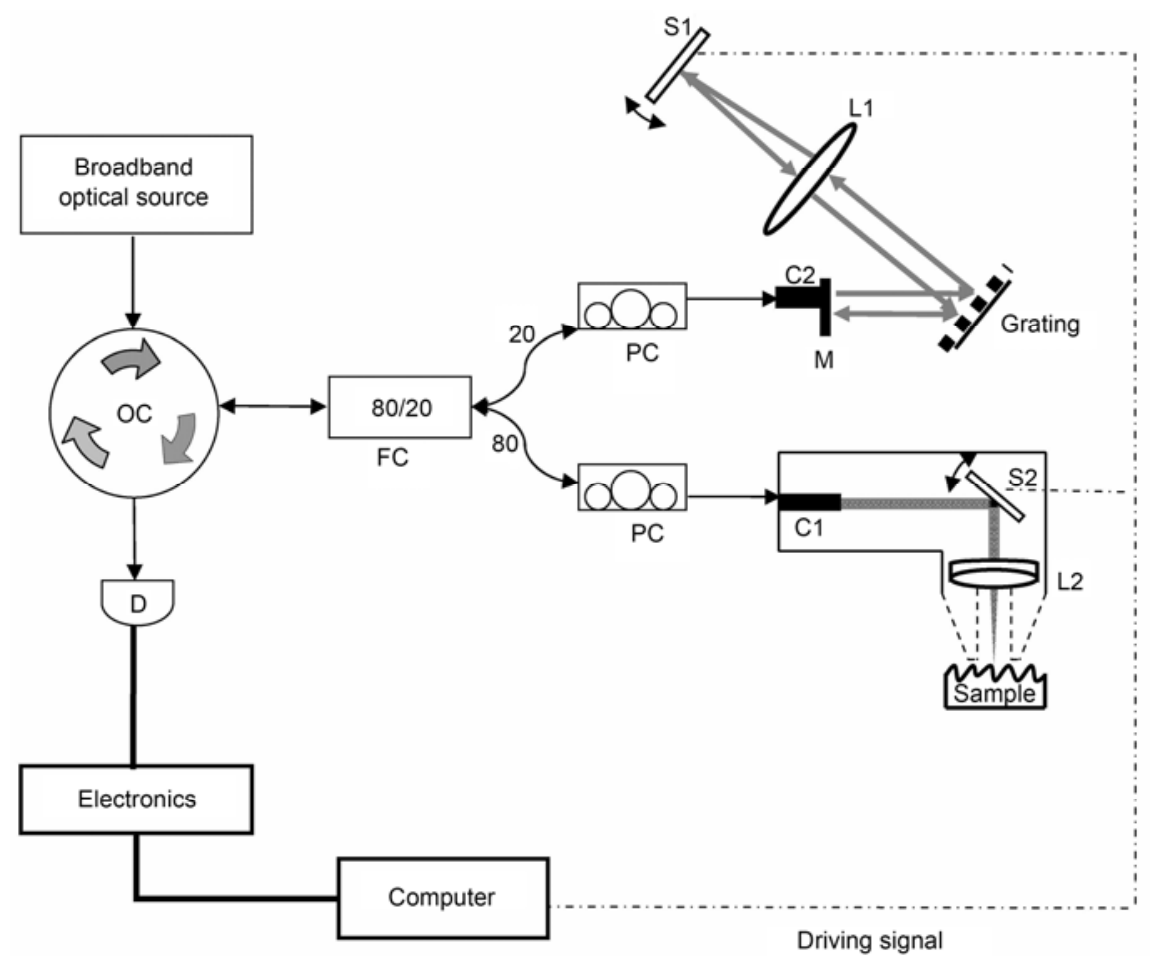

Figure 1 Setup of the handheld optical coherence tomography system. FC, fiber coupler; PC, polarization controller; C1 and C2, collimators; L1 and L2, lenses; S1 and S2, optical scanners; D, detector; OC, optical circulator; M, end mirror. 
$10 \mu \mathrm{m}$ in air, the resolution is $10 / 1.35 \approx 7 \mu \mathrm{m}$ in tissue. Only one focusing lens is used in the handheld device to reduce reflections and the corresponding optical loss. In contrast to a traditional RSOD, the end mirror (M) and input fiber collimator $(\mathrm{C} 1)$ are placed on the same mirror mount. This will ensure that the end mirror is kept perpendicular to the light coming out of the collimator. Because of this patented design, the system is much less sensitive to environmental variations and RSOD [14]. Moreover, it is also much more stable and easier to adjust. The focal lens (L1) and optical scanner (S1) were placed on a scanning stage. Therefore, the dispersion can be easily adjusted and the system sensitivity can be optimized by scanning the stage.

\section{Clinical analysis}

Human skin is composed of the epidermis (0.04-0.16 mm thick) and the dermis. The dermis is divided into papillary and reticular layers. PDT treatments vary because of the range of thicknesses of the epidermis and vascular distributions in the dermis papillary layer. For a thick epidermis and dense vascular distribution, a large laser radiation dosage is needed and the laser wavelength should also be taken into account. An image of the cross-sectional structure of the patient's skin is very helpful in clinical analysis. In particular, images with resolutions sufficient to distinguish the dilated vascular capillaries of the patient are needed for this process.

We performed OCT imaging using our handheld probe system. The images, obtained using the system, clearly resolve the cross-sectional dermal structure of the skin sample (Figure 2). The epidermis, papillary layer and dermis can be distinguished in the image shown in Figure 2(a). The thickness of the epidermis can be measured by the optical path length of the OCT image. This can aid in optical dosage selection for PDT. Another factor critical for setting the optical dose is the vascular distribution, which can be seen in the images shown in Figure 2(b) and (c). Dark areas can be seen in Figure 2(b) and (c), and are indicated by the arrows. The diameter of these areas varies between about $80-110 \mu \mathrm{m}$ and they are more than $100 \mu \mathrm{m}$ deep. By rotating the handheld probe by a small angle about the axis perpendicular to the surface, the shape of the dark area (circled) changes from an oblong (Figure 2(b)) to circular (Figure 2(c)). It can be seen that the OCT image in Figure 2(b) was taken along the orientation parallel to the blood vessel. Therefore, the dark area looks like a tube. However, Figure 2(c) is perpendicular to the vessel's orientation. Therefore, the dark area looks like a round hole. Thus, the circled dark area is a blood vessel.

Comparing the OCT image with the skin biopsy [1] shown in Figure 3, the dark areas in the OCT image have the same diameter and hypodermic depth in the papillary layer of the corium as the dilated capillary vessels seen in
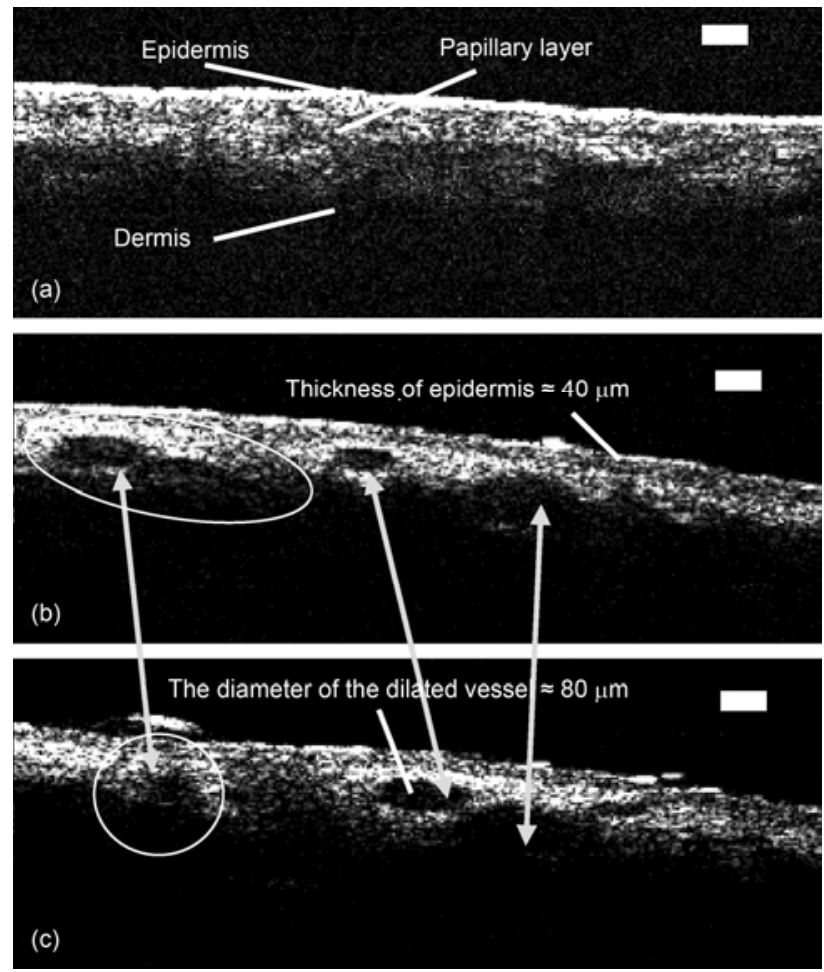

Figure 2 OCT image of normal skin (a), PWS skin (b) and the same PWS skin sample for a different orientation angle of the handheld probe (c). Scale bar: $100 \mu \mathrm{m}$.

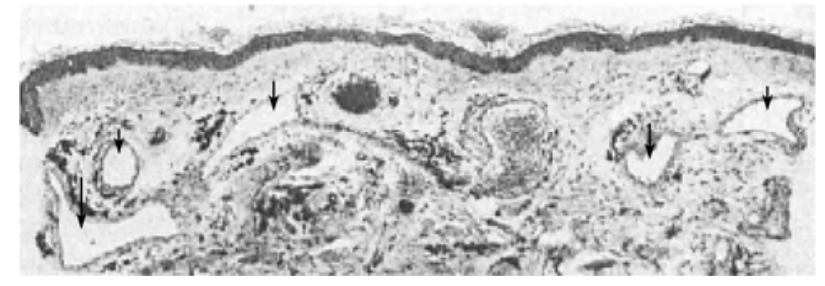

Figure 3 Skin biopsy from patient with PWS. The distended capillaries are marked with arrows. The vessel depth ranges from 290 to $630 \mu \mathrm{m}$.

the skin biopsy. The diameter, configuration and depth all coincide with the dilated capillary vessels. Therefore, we conclude that the cylindrical tissue seen in the OCT image corresponds to vascular malformations [2].

Furthermore, we can measure the thickness of the epidermis and the papillary layer. Depending on the diameter and configuration of the dilated capillary vessels, doctors can adjust the dosage, laser intensity and treatment time, accordingly. The diameter of capillary vessels in normal skin is about $10 \mu \mathrm{m}$. Therefore, normal vascular structures cannot be clearly imaged with the resolution ( $8 \mu \mathrm{m}$ (lateral) $\times 7 \mu \mathrm{m}$ (axial)) available in our OCT system. However, the diameters of dilated capillary vessels in patients with PWS are usually much larger, typically $50-100 \mu \mathrm{m}$. These capillaries would be large enough to be visible in OCT images.

Typically, a procedure begins with the injection of a 
photosensitizer (HMME [3]). Glycerin will also be applied to the affected area to increase skin penetration. After $5 \mathrm{~min}$, OCT imaging will be performed on the affected area of the patient. Then the patient will be exposed to a laser beam for more than $30 \mathrm{~min}$ (532 $\mathrm{nm} \mathrm{CW}$ laser). The dissociation product of the photosensitizer, which is generated by laser radiation, destroys the capillary lesions without any damage to the skin. When laser operation ceases, the affected area will be imaged again using OCT in situ. With the handheld probe, we are able to ensure that the two imaging sessions occur at the same location through the use of a pre-marker. We can then cross reference these two OCT images to characterize the effectiveness of the treatment. It was found that there is little change in the dermal structure before and immediately following the treatment. This implies that PDT involves relatively slow processes. The capillary lesion is destroyed, but it may take nearly two weeks for the macrophages to collect and ingest the remnants of the capillary [15]. Therefore, the patient would need a month to recover before the next treatment. OCT imaging can provide a very good cross reference for each PDT session and a direct assessment of the therapy via the change in vascular malformation at any point after the treatment. In fact, patients may need 10 sessions of PDT to completely remove the discoloration. Although the effects of only one therapy session cannot be seen through OCT imaging, the change in the dermal structure can be seen in OCT images after several therapy sessions. Therefore, OCT is a good tool for assess- ment after a series of therapies.

Furthermore, comparing OCT images of the same patient for normal and affected areas provides doctors with a good cross reference for clinical diagnosis and surgical guidance. Note that the OCT image of the normal area is made at the corresponding spot of the contra lateral normal skin. OCT can supply high-resolution information on the dermal structure, which is critical for clinical diagnosis and helpful for treatment selection.

Figures 4 and 5 show the photographs and OCT images of two patients. As expected, vascular malformations are observed in the OCT images of the affected areas, but no distended capillaries are observed in the OCT images of the normal area. These results were consistent for 28 of the 30 patients who participated in the clinical operation. These vascular malformations are responsible for the reddish (show as dark grey area in Figures 4 and 5) discoloration of the skin. Generally, doctors select treatment based on the deepness of the discoloration. This is based on the assumption that the darker the PWS, the more severe the condition. From the photographs, it can be seen that the red stain on the second patient (Figure 4) is darker than that of the first one (Figure 5). Therefore, the second patient would be exposed to a longer laser treatment on the assumption that his condition was more severe. However, the opposite is true. OCT imaging shows that the depth of the second patient's dilated vessel network is $200-300 \mu \mathrm{m}$, which is less than that of the first patient's dilated vessel network $(300-400 \mu \mathrm{m})$.

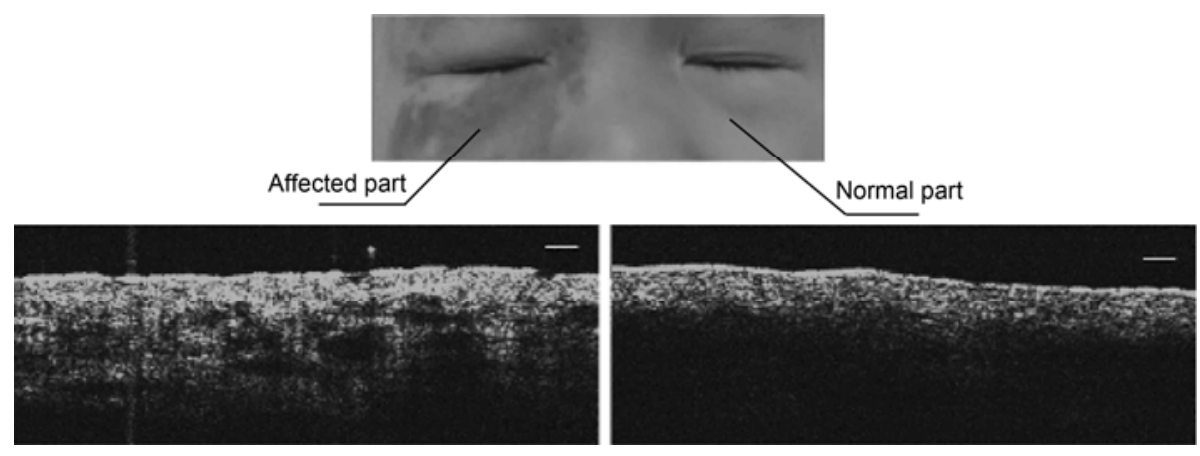

Figure 4 Clinical OCT images from patient No. 1. Scale bar: $100 \mu \mathrm{m}$.

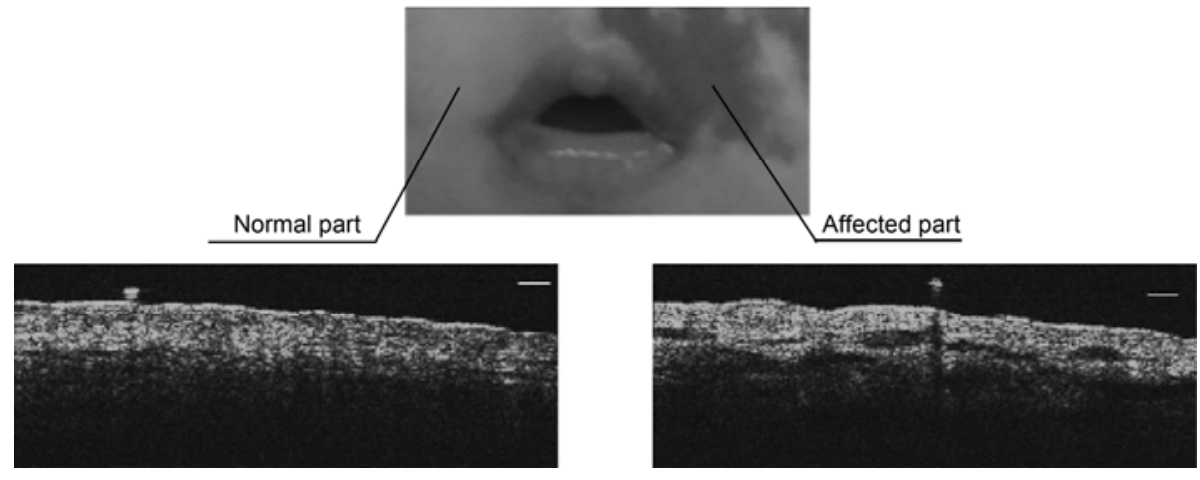

Figure 5 Clinical OCT images from patient No. 2. Scale bar: $100 \mu \mathrm{m}$. 
This implies that the second patient's condition appears worse than it really is. However the first patient's dilated vessel network is more concentrated than the second one's. The dilated vessels of both patients are similar in size $(80 \mu \mathrm{m}$ in diameter). Therefore, the difference in the color is because of the depth and distribution rather than size of the blood vessels. Therefore, according to the OCT images, the first patient needs a longer laser treatment than the second one because his dilated vessels are located deeper in the skin and are more concentrated. Using the OCT images, the doctor can select more effective treatments for a patient. Note that OCT also shows promise for use in PDT assessments comparing images before and after the operation. However, to make a good comparison, the two images should be taken at exactly the same location or within the OCT spatial resolution, which is less than 10 microns. One possible solution is to take a $3 \mathrm{D}$ image of a volume of tissue before and after the therapy. Then it is easier to locate the same part of the tissue to facilitate comparison. Nevertheless, this study shows that OCT provides information that is useful in clinical diagnosis.

\section{Conclusions}

An OCT system with a handheld probe, an imaging speed of 4 frames per second, an array of $400 \times 400$ pixels, a spatial resolution of $8 \mu \mathrm{m}$ (lateral) $\times 10 \mu \mathrm{m}$ (axial) and a sensitivity that can be as high as $102 \mathrm{~dB}$ was used for real-time imaging PWS patients. The data collected were used to guide and assess the PDT treatment selected for the patient. OCT imaging provides useful information on the dilated capillary vessels and thicknesses of the epidermis and papillary layer. With the aid of OCT, the doctor can make a more accurate pathological diagnosis and select a therapy that is more appropriate for the patient. In addition, OCT images are a useful tool to assess the effectiveness of the treatment. OCT is a promising new technology with clinical applications to dermatology and related areas.
The authors would like to thank the New Raysing Company for their technical support. This work was supported in part by the National High-Tech Research and Development Program of China (2006AA02Z472), National Basic Research Program of China (001CB510307 and 2009CB929400), National Natural Science Foundation of China (90508001, 10676014 and 10574081) and Tsinghua University Initiative Scientific Research Program.

1 Barsky S H, Rosen S, Geer D E, et al. A computer-assisted study. J Invest Derm, 1980, 74: 154-157

$2 \mathrm{Gu} \mathrm{Y,} \mathrm{Li} \mathrm{J} \mathrm{H,} \mathrm{Guo} \mathrm{Z} \mathrm{H,} \mathrm{et} \mathrm{al.} \mathrm{Port} \mathrm{wine} \mathrm{stain} \mathrm{treated} \mathrm{by} \mathrm{photodynam-}$ ic therapy (in Chinese). Beijing Med J, 1991, 13: 317

3 Wang Y, Gu Y, Liu F G, et al. Photo bleaching reaction kinetic rules of HMME in different solutions (in Chinese). Chin J Laser Med Surg, 2007, 16: 73-78

4 Zhang J C, Liu W, Yi Z Z, et al. The molecular mechanism of photodynamic therapy to fibrosis: Regulation on the pyridinoline crosslink formation in collagen. Chin Sci Bull, 2009, 54: 2230-2234

5 Xu S J, Zhang X X, Chen S, et al. Novel phototherapeutic agents: Investigation and progress of hypocrellin derivatives. Chin Sci Bull, 2003, 48: 1775-1785

6 Chen Y W, Li L, Zhang M H, et al. An explanation to the high efficiency of m-THPC (tempor-fin) used in photodynamic therapy. Chin Sci Bull, 2001, 46: 823-827

7 Jiang L J, He Y Y. Photophysics, photochemistry and photobiology of hypocrellin photosensitizers. Chin Sci Bull, 2001, 46: 6-16

8 Zhao P D, Chen P, Tang G Q, et al. Two-photon spectroscopic behaviors and photodynamic effect on the BEL-7402 cancer cells of the new chlorophyll photosensitizer. Sci China Ser B-Chem, 2008, 51: 529-538

9 Dong H Q, Zhao Z L, Wen H Y, et al. Poly(ethylene glycol) conjugated nano-graphene oxide for photodynamic therapy. Sci China Chem, 2010, 53: 2265-2271

10 Li Z B, Wang J G, Chen J R, et al. Hypocrellin B doped and pHresponsive silica nanoparticles for photodynamic therapy. Sci China Chem, 2010, 53: 1994-1999

11 Huang D, Swanson E A, Lin C P, et al. Optical coherence tomography. Science, 1991, 254: 1178-1181

12 Drexler W, Morgner U, Kartner F X, et al. In vivo ultrahigh-resolution optical coherence tomography. Opt Lett, 1999, 24: 1221-1223

13 Xue P, Fujimoto J G. Ultrahigh resolution optical coherence tomography with femtosecond Ti:sapphire laser and photonic crystal fiber. Chin Sci Bull, 2008, 53: 1963-1966

14 Tearney G J, Bouma B E, Fujimoto J G. High-speed phase- and group-delay scanning with a grating-based phase control delay line. Opt Lett, 1997, 22: 1811-1813

15 Zhao S Y, Gu Y, Xue P, et al. Imaging port wine stains by fiber optical coherence tomography. J Biomed Opt, 2010, 15: 036020

Open Access This article is distributed under the terms of the Creative Commons Attribution License which permits any use, distribution, and reproduction in any medium, provided the original author(s) and source are credited. 